\title{
Explore the Relationship between Insulin Resistance and PGC1 $\alpha$ in PCOS Mice
}

\author{
Lei Chen ${ }^{1 \#, ~ X i n ~ T a o ~}{ }^{* \#}$, Shuqi Ge ${ }^{2}$, Lisi Cai ${ }^{1}$, Xuanying Deng ${ }^{1}$ \\ ${ }^{1}$ Center for Reproductive Medicine, The Third Affiliated Hospital, Sun Yet-sen University, Guangzhou, China \\ ${ }^{2}$ Department of Infertility and Sexual Medicine, The Third Affiliated Hospital, Sun Yet-sen University, Guangzhou, China \\ Email:*taoxdoctort@163.com
}

How to cite this paper: Chen, L., Tao, X., Ge, S.Q., Cai, L.S. and Deng, X.Y. (2018) Explore the Relationship between Insulin Resistance and PGC1 $\alpha$ in PCOS Mice. Open Journal of Endocrine and Metabolic Diseases, 8, 71-80.

https://doi.org/10.4236/ojemd.2018.83008

Received: January 28, 2018

Accepted: March 13, 2018

Published: March 16, 2018

Copyright (๑) 2018 by authors and Scientific Research Publishing Inc. This work is licensed under the Creative Commons Attribution International License (CC BY 4.0).

http://creativecommons.org/licenses/by/4.0/

\begin{abstract}
Objective: To investigate the relationship between AMPK $\alpha$, PGC $\alpha$, insulin resistance and reproductive function in PCOS mice and to find out the molecular mechanisms and potential therapeutic molecular targets of pathogenesis of PCOS. Methods: The PCOS mouse model was established by DHEA administering with Balb/c mice. And after AMPK agonist AICAR and inhibitor Compound $\mathrm{C}$ intervention, Fasting blood glucose, fasting insulin and testosterone levels were observed. The HOMA index was calculated. The changes of PGC $\alpha$ expression in ovarian tissue were observed by western blot and immunohistochemistry to determine the relationship between insulin resistance and PGC $\alpha$ in PCOS mice. Results: Western blot and immunohistochemistry showed that PGC1 $\alpha$ protein was expressed in the ovary of mice and the expression of PGCl $\alpha$ was negatively correlated with AMPK $\alpha$ in our study. Compared with the control group, the expression of PGC1 $\alpha$ in the ovaries of the mice in PCOS group was significantly increased $(\mathrm{P}<0.05)$, after intervention with AMPK agonist AICAR, the expression of PGC1 $\alpha$ in PCOS + AICAR group was lower than that in PCOS group $(\mathrm{P}<0.05)$. It is worth noting that the expression of PGC1 $\alpha$ in PCOS mice exposed to AMPK inhibitor Compound $\mathrm{C}$ also decreased compared with $\mathrm{PCOS}$ group $(\mathrm{P}<0.05)$. Conclusion: In ovarian tissue, the insulin resistance-related AMPK $\alpha$ pathway in PCOS mice may be negatively correlated with PGC $\alpha$.
\end{abstract}

\section{Keywords}

PCOS, Mice, Insulin Resistance, PGC1 $\alpha$

\section{Introduction}

Polycystic ovary syndrome (Polycystic ovary syndrome, PCOS) is one of the

\#The authors contributed equally to this work. 
most common endocrine and metabolic disorders in women of childbearing age with a prevalence of $8.3 \%-9.13 \%$ [1]. It is the main cause of anovulatory infertility, characterized by polycystic ovary changes, menstrual disorders and hyperandrogenism. PCOS patients are often accompanied by insulin resistance (IR), and IR is closely related to reproductive dysfunction such as infertility and habitual abortion [2]. However, it remains unclear how insulin resistance influence the reproductive and metabolic functions of women with PCOS.

Peroxisome proliferator-activated receptor $\gamma$ coactivator $1 \alpha(\mathrm{PGCl} \alpha)$, a nuclear transcriptional coactivator that regulates multiple biological processes such as mitochondrial biogenesis, fatty acid oxidation and glucose metabolism, is also associated with fatty acid metabolism and insulin sensitivity. Studies have shown that insulin resistance occurs in skeletal muscle cells of rats in a high glucose environment induced by down-regulation of the AMP activated protein kinase (AMPK)/PGC- $1 \alpha$ signaling pathway [3], the protective effects of AMPK/PGC-1 $\alpha$ pathway were found in the kidney tissues of type 2 diabetic mice [4], peripheral nerves of diabetic rats and skeletal muscle cells [5]. However, there are no studies exploring PGC1 $\alpha$ molecules in ovarian tissue of PCOS.

PCOS patients are often accompanied by IR; our preliminary study has found the protective effects of AMPK $\alpha$ molecule in PCOS mice with IR [6], from this we speculate that PGC1 $\alpha$ plays an important role in the pathogenesis of IR in PCOS. Based on the above assumptions, we conducted an in vivo PCOS mouse experiment to investigate the association between insulin resistance-related AMPK $\alpha$ pathway and PGC $\alpha$ in PCOS mice and to find out more molecular mechanisms and potential therapeutic targets for the pathogenesis of PCOS.

\section{Materials and Methods}

\subsection{Experimental Subjects}

Forty Balb/c (25 days old) female mice provided by the Experimental Animal Center of Sun Yat-sen University in Guangzhou, China (SCXK (Guangdong): 2016-0029). The animals were of specific pathogen free (SPF) grade, with a mean body mass is $12.925 \pm 1.515 \mathrm{~g}$.

\subsection{Methods}

\subsubsection{The Main Reagent}

$\beta$-actin primary antibody was purchased from Cell Signaling Technology (CST) (3700). PGC1 alpha primary antibody was purchased from Abeam (ab54481). Dehydroepiandrosterone (DHEA) was purchased from Merck (252805), Compound C was purchased from Selleck (S7306) and AICAR was purchased from Santa Cruz (sc-200659). EnVision +/HRP kit is a production of Beijing ZSGB-BIO ORIGENE.

\subsubsection{Ethical Support}

All the mice involved in the experiment are strictly in accordance with "The guidelines of Sun Yat-sen experimental animal care and use", the program was 
approved by the Animal Care and Use Committee of the Third Hospital of Sun Yat-sen University.

\subsubsection{PCOS Mouse Model Establishment and Reagent Intervention}

Forty female BALB/c mice ( 25 days old) were randomly divided into two groups: control group $(n=9)$ and PCOS group $(n=31)$, which were all fed in the center of Sun Yat-sen Guangdong (2012-0081) with constant room temperature of $22^{\circ} \mathrm{C}$ and air humidity of $55 \%$. The PCOS group was given DHEA $(6 \mathrm{mg} / 100 \mathrm{~g}$ body weight in $0.1 \mathrm{ml}$ of soybean oil) via hypodermic and the control group was given saline $(0.1 \mathrm{ml})$ by the same method. Both groups were injected for 20 days. Vaginal secretions were smeared on every morning from the 11th day and detected under the microscope.

Mice in the PCOS model group lost their regular estrous cycles and the model was considered successfully established [6]. Four PCOS mice and two control groups were randomly selected for fasting serum testosterone (T), fasting insulin (FINS), fasting plasma glucose (FGLU) detection, HOMA-IR index calculation and the ovarian HE staining was use to further assess the efficiency of model creation, the results of blood testing showed that: after pretreatment with DHEA for 20 days, the PCOS group was in an apparently insulin resistant state $(\mathrm{P}=$ $0.036)$ and serum testosterone was significantly elevated $(P=0.014)[6]$. The remaining mice in the PCOS model group were randomly divided into the PA $(\mathrm{PCOS}+$ AICAR $)$ group $(\mathrm{n}=10)$, the $\mathrm{PC}(\mathrm{PCOS}+$ compound $\mathrm{C})$ group $(\mathrm{n}=10)$ and the PCOS group $(n=7)$. The rats in PA group were injected intraperitoneally with $50 \mathrm{mg} \cdot \mathrm{kg}^{-1} \cdot \mathrm{d}^{-1}$ of AICAR dissolved in $0.2 \mathrm{ml}$ sterile distilled water. The PC group was injected through tail vein with $10 \mu \mathrm{g} \cdot \mathrm{kg}^{-1} \cdot \mathrm{d}^{-1}$ compound C dissolved in $0.1 \mathrm{ml}$ sterile distilled water. PCOS and control group $(n=7)$ were given $0.2 \mathrm{ml}$ sterile distilled water by intraperitoneal injection. All injections lasted 7 days, and all mice were given a normal diet daily.

\subsubsection{Blood Samples and Ovarian Tissue Acquisition, Modeling and Testing the Effect of Intervention}

Seven days after the intervention, the mice were fasted for 12 hours, then we cut off the tail tip of the mice to take blood drop to test fasting blood glucose. Then eyeballs were removed to obtain blood. Fresh blood was allowed to stand at $37^{\circ} \mathrm{C}$ for 30 minutes and then centrifuged to obtain a supernatant. Serum was stored at $-80^{\circ} \mathrm{C}$ and used again for the subsequent hormone assay. $\mathrm{T}$ and FINS levels were measured with a radioimmunoassay kit (Beijing Institute of Biotechnology, Beijing, China); FGLU was measured with a blood glucose meter and test paper (BRAUN, Germany). And HOMA-IR was calculated to assess the IR degree. Previous results have showed that compared with PCOS group, the insulin sensitivity of the mice in the PA group improved (HOMA-IR $=7.621 \pm 1.66$ vs $10.133 \pm 1.654, \mathrm{P}<0.05)$ and the estrous cycle became regular after 7 days of AICAR treatment, however, PCOS group and PC group are still in a status of insulin resistance (HOMA-IR $=10.765 \pm 2.036$ vs $10.133 \pm 1.654, \mathrm{P}<0.05)$ after 7 days of Compound $\mathrm{C}$ treatment. In addition, androgen levels did not recover 
significantly [6].

Ovaries were quickly removed and fixed in $4 \%$ paraformaldehyde immediately after blood samples were collected. Ovaries were routinely dehydrated 24 hours after formalin fixation and then were paraffin-embedded and sectioned $(4 \mu)$ for HE staining to observe the ovarian tissue structure. Morphological changes in ovarian tissue specimens were examined by optical microscopy. In the control group, microscopic examination revealed the presence of follicles of different stages and a few corpora lutea; granulosa cells were orderly arranged in an intact form, mostly in 4 - 6 layers [6].

Each sample plus $300 \mu \mathrm{L}$ protein lysis solution, respectively, with a 5 -mL tissue homogenizer were used to ablated ovarian tissue, the lysate was centrifuged for $15 \mathrm{~min}, 4^{\circ} \mathrm{C}$ at $3000 \mathrm{BPM}$. The supernatant fractions were collected and centrifuged for $15 \mathrm{~min}, 4^{\circ} \mathrm{C}$ at $3000 \mathrm{BPM}$. The protein content was determined using a microbicinchoninic acid assay (Pierce, Rockford, IL) using bovine serum albumin (BSA) as a standard.

After Sodium dodecyl sulfate-polyacrylamide Gel (SDS-PAGE Gel) was prepared, protein samples were loaded directly into SDS-PAGE gel sample wells, 20 $\mu \mathrm{l}$ per well, $2 \mu \mathrm{l}$ Maker points at the far left. Add ice in the basin and set the voltage at $100 \mathrm{~V}$. Electrophoresis is stopped when the bromophenol blue reaches the bottom of the gel.

Place into the clip in turn according to the order in turn of sponge, filter paper, plastic, membranes (wet in methanol first), filter paper, sponge, inserted into the membranes slot (160 mA, $90 \mathrm{~min}$; Ice basin). When transfer membranes is completed, stained with Pichun red dye for two minutes to verify whether the protein was transferred to the membrane, cleaned in methanol. Cut down $\beta$-actin $(47 \mathrm{KDa}), \mathrm{AMPK} \alpha 1 / 2(62 \mathrm{KDa})$, and placed them in a pre-prepared TBST incubator (Shaker, 2 mins). The blotted membranes were blocked in PBS containing 5\% milk (2 g Milk, $40 \mathrm{ml}$ TBST) for $1 \mathrm{~h}$ at room temperature.

The blotted membranes were then incubated and reacted overnight with 1:1,000-diluted primary antibody (AMPK and $\beta$-actin) in TBS containing 5\% BSA at $4{ }^{\circ} \mathrm{C}$. After being washed with three times of TBS containing $0.1 \%$ Tween 20 , the blotted membranes were incubated and reacted with 1:2,000-diluted peroxidase-conjugated secondary antibody in TBS containing 5\% BSA for $1 \mathrm{~h}$ at room temperature.

After the membranes were washed with four times of TBS containing $0.1 \%$ Tween 20, Lumi GLO from a Phototope-HRP Western Detection Kit was added to the blotted membranes and reacted for $1 \mathrm{~min}$. The membranes were then covered with plastic wrap and exposed to X-ray film for 1 to $2 \mathrm{~min}$. The treated membranes were laid on a blue plate and Exposure with Tanon 5200 imager. These experiments were repeated two times.

Previous results showed that: PCOS mice ovarian AMPK $\alpha$ protein expression decreased, AICAR intervention increased AMPK $\alpha$ protein expression, and AMPK $\alpha$ protein expression in Compound C intervention is still low [6]. 


\subsubsection{Immunohistochemical (IHC) Detection of Mouse Ovarian Tissue PGC1 $\alpha$ Molecular Protein Expression}

The EnVision method was used for IHC staining. Sections (5 - $7 \mu \mathrm{m}$ thick) were cut following a conventional procedure. The section specimens were affixed to silicate glass and heated at $56^{\circ} \mathrm{C}$ for $1 \mathrm{~h}$. After dewaxing in fresh xylene $(5 \mathrm{~min} \times$ 3 times), the specimens were treated with gradient ethanol dehydration $(100 \%$, $100 \%, 90 \%, 80 \%, 70 \%$ and 50\%, 5 min each) and rinsed with tap water, followed by two rinses with distilled water (each for $5 \mathrm{~min}$ ). After the slices were drilled, the tissue was circulated around the tissue with a group pen (to prevent antibody flow away), and the tissue was covered with $5 \%$ horse serum uniformly. The tissue were placed in a wet box at $37^{\circ} \mathrm{C}$ for $1 \mathrm{~h}$ and the rinsed specimens were fixed with pH 8.0 ethylenediaminetetraacetic acid (EDTA) under high pressure and then cooled for $20 \mathrm{~min}$. After rinsing with phosphate-buffered saline (PBS) for three times (each for $5 \mathrm{~min}$ ), $50 \mu \mathrm{L}$ of primary antibody PGC1 $\alpha$ (dilution 1:250) was added to each specimen and incubated in a humidified chamber at $37^{\circ} \mathrm{C}$ for overnight. After placed at room temperature for $15 \mathrm{~min}$ to rewarm the temperature, pipette recovery of primary antibody, PBS wash for 3 times, each for $5 \mathrm{~min}$. The rinsed specimens were placed in 3\% hydrogen peroxide for microwave heating at low power for $3 \mathrm{~min}$ and then rinsed with tap water, followed by two rinses with distilled water (each for $5 \mathrm{~min}$ ).The specimens were rinsed with PBS for three times (each for $5 \mathrm{~min}$ ), and each specimen was added with drops of secondary antibody and then incubated in a humidified chamber at $37^{\circ} \mathrm{C}$ for $1 \mathrm{~h}$. After washing with PBS for three times (each for $5 \mathrm{~min}$ ), a 3, 3'-diamino benzidine (DAB) solution (1:20) was added to the specimens for coloration, and the reaction was terminated with tap water. Hematoxylin was used for counterstaining, and tap water was used for bluing. The specimens were dried and mounted for microscopic examination and photographing. A negative control was prepared by substituting PBS for the primary antibody.

\subsubsection{Statistical Analysis}

The statistical analysis was performed using SPSS 22.0 (SPSS Inc., Chicago, IL, USA). The measurement data were expressed as the mean \pm standard deviation (SD). A normality test and homogeneity of variance test were performed prior to comparison of the group means. An independent two-sample $t$ test was used for homogeneity of variance, and the $t$ test was used for heterogeneity of variance. Variables that did not meet normal distribution were analyzed using a non-parametric test. Comparisons of multiple samples were performed using one-way ANOVA at the 0.05 level. A P value of $<0.05$ was considered statistically significant.

\section{Results}

\subsection{HE Staining Results after Modeling}

Twenty days after DHEA intervention, ovarian tissue was made into paraffin sections for HE staining. The results (Figure 1) show that, in the control group, 
microscopic examination revealed the presence of follicles of different stages and a few corpora lutea; granulosa cells were orderly arranged in an intact form, mostly in 4 - 6 layers. In the PCOS group, oocytes or corona radiation were observed within the follicles, and follicles of different stages and corpora lutea were rare in the cortex. Furthermore, the number of immature small follicles was significantly increased, and granulosa cells were arranged loosely in fewer (only 1 3) layers, with atresia of some follicles being observed.

\subsection{Western Blot Results}

The expression of PGC1 $\alpha$ protein in ovarian tissue of PCOS group was significantly increased $(\mathrm{P}<0.05)$, and the expression of $\mathrm{PGC1} \alpha$ protein was down-regulated by AICAR $(\mathrm{P}<0.05)$ (Figure 2, Figure 3$)$. It is noteworthy that the expression of PGC1 $\alpha$ in PCOS mice treated with AMPK inhibitor Compound $\mathrm{C}$ was also lower than that in PCOS mice $(\mathrm{P}<0.05)$. Although the expression of PGC1 $\alpha$ was slightly higher in the inhibitor group than in the stimulation group, the difference is not obvious (Figure 2, Figure 3). This inconsistency may be explained by the fact that there may be other molecular linkages between these two molecules in the hyperandrogenic environment and that PCOS mice may have some kind of stress response or adaptive regulation in their own bodies or may be associated with the heterogeneity related to individual responses between mice.

(a) Control group

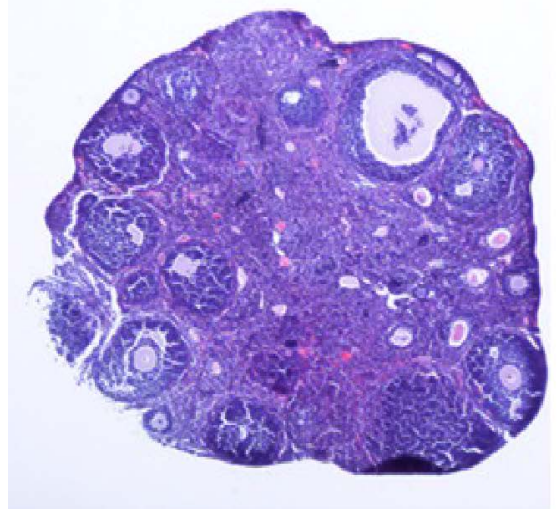

(b) PCOS group

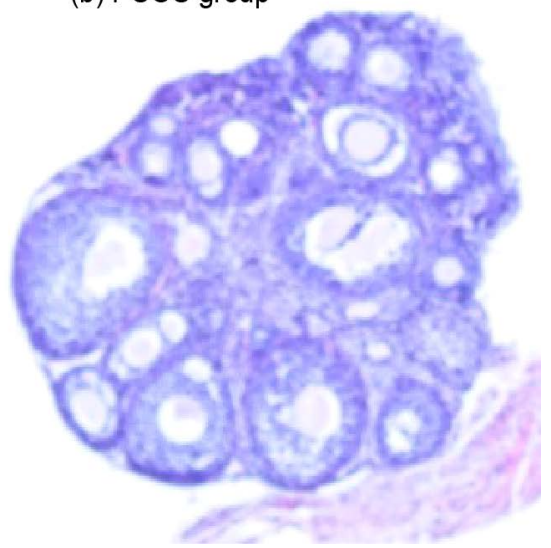

Figure 1. The ovarian HE staining of the two groups of mice after DHEA intervention.

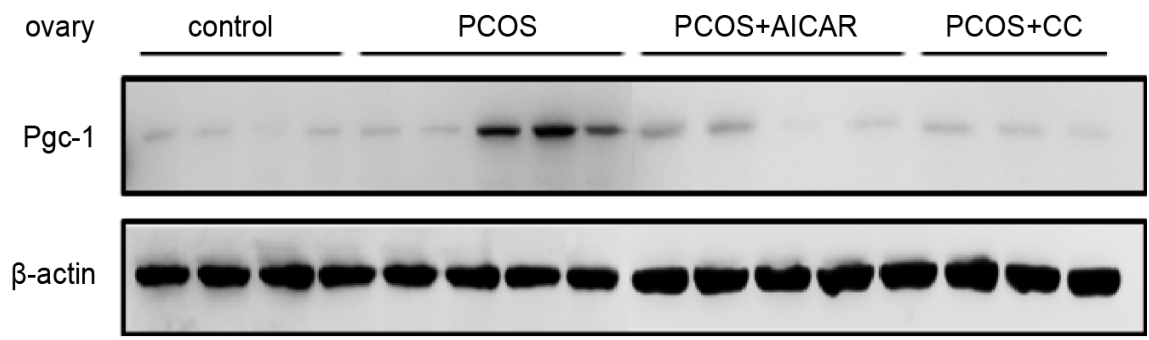

Figure 2. Comparison of the expression of PGC1a in ovaries of balb/c mice between 4 groups. 


\subsection{Immunohistochemistry Results}

Immunohistochemical detection of PGC1 $\alpha$ protein expression was consistent with western blot results. The e PGC1 $\alpha$ protein expression of PCOS group was significantly increased, and AICAR intervention down-regulated PGC1 $\alpha$ protein expression (Figure 4). However, due to the short intervention period, ovarian histology has not shown any significant improvement even as serum hormone levels and reproductive function began to improve (Figure 4).

\section{Discussion}

PCOS is a female endocrine disorder characterized by polycystic ovary changes, menstrual disorders and hyperandrogenism. In the hyperandrogenic environment, follicular development is blocked, leading to atresia of the follicle. High levels of androgens may also cause long-term damage to insulin sensitivity [7].

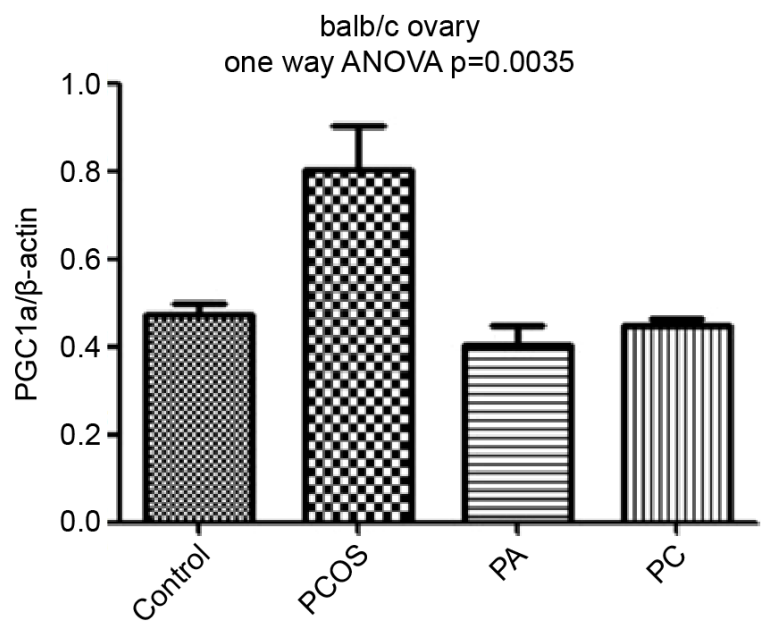

Figure 3. Comparison of the expression of PGCla in ovary of balb/c mice between four groups.

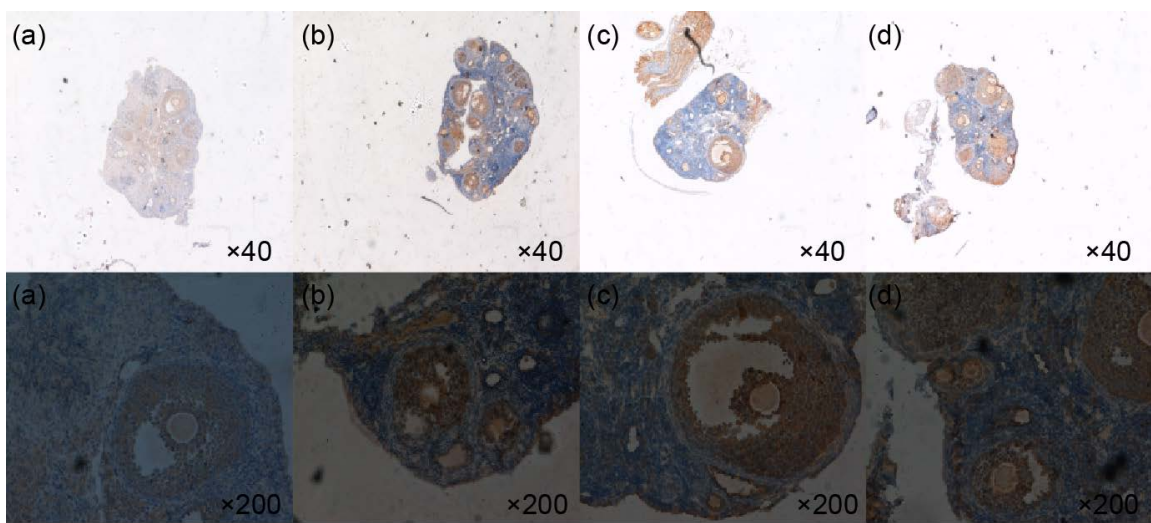

Figure 4. Comparisons of expression of PGCla in ovary of four groups. (a) Control group; (b) PCOS group; (c) PCOS + AICAR group; (d) PCOS + CC group. The expression of PGC1a protein in PCOS group was lower than that in control group, in PCOS + AICAR group the expression of PGCla was increase, however, the application of CC continue to inhibit he expression of PGC1a, in addition. 
AMPK is the core of cellular energy metabolism, is the hub of cellular signaling. It is considered as a cell's "energy sensor" and the activated AMPK can increases intracellular ATP stores by enhancing catabolism and inhibiting anabolism when cell nutrition and energy are insufficient. Many studies found that AMPK is closely related to glucose and lipid metabolism, inflammation and insulin resistance. AMPK agonists such as metformin and AICAR improve endocrine and reproductive function in PCOS [8] [9]. Our previous experiments also found that AICAR intervention can improve the endocrine disorders in PCOS mice [6].

PGC1 $\alpha$ regulates multiple biological processes such as mitochondrial biogenesis and fatty acid oxidation [10]. A study found that increased PGC1 $\alpha$ activity can improve mitochondrial function and IR in skeletal muscle [11]. In an in vitro study of skeletal muscle cells, AMPK directly phosphorylates PGC1 $\alpha$ and promotes its transcription [12]. We know that mitochondrial is one of the most important organelles in the energy metabolism. The above evidence proves that PGC1 $\alpha$ plays an important role in maintaining mitochondrial function. However, the elevated PGC1 $\alpha$ expression in ovarian tissue of PCOS mice in our study suggests that mitochondria in ovarian cells of PCOS mice are active, indicating that the oxidative status of fatty acids in the ovarian tissue is excessive active. However, after upregulated AMPK $\alpha$ expression with ACIAR, we observed decreased PGC1 $\alpha$ protein expression in ovarian tissue and improved PCOS insulin resistance. Previous studies have found that in adipocytes, fibroblast growth factor 21 can enhance mitochondrial function and fat oxidation by activating AMPK, SIRT1 and PGC1 $\alpha$ molecules [13]. In peripheral nerve tissue, upregulation of AMPK-PGC1 $\alpha$-SIRT1 pathway can promote glucose metabolism and thus protect peripheral nerve function in diabetic rats [5]. However, in our study, we found a new finding in comparison with the previous studies. We found that the insulin resistance-related AMPK $\alpha$ pathway in PCOS mice is negatively correlated with the PGC $\alpha$ molecule. From this we make the following conjecture: Glucose metabolism disorder, could also cause the fatty acid oxidation rise in order to maintain the energy supply. Glucose metabolism disorders lead to excessive accumulation of more fat than oxidative metabolism, energy metabolism in general is a negative balance. However, there was no difference in body weight among our groups. This result may be related to the small body weight of mice and the short time of intervention. PGC1 $\alpha$ expression may be in a tissue-specific manner in the regulation of different diseases [14], and we hypothesize that PGC1 $\alpha$ expression may also be associated with hyperandrogenism. PCOS is a complex endocrine disorder, there is usually hyperandrogenism in vivo which will severely impaired glucose tolerance [15], PCOS patients often associated with impaired glucose tolerance, and pathological changes of PCOS are mainly located in the ovary, Previous studies have not explore PGC1 $\alpha$ in ovarian tissue of PCOS. 


\section{Limitation}

Some of the limitations of our study are: Our sample size is small, and the study was not an intervention with drugs directly, but only intervened on protein level, and the intervention is not long enough. In addition, the dose of DHEA we used is far exceeds the blood concentration of human PCOS patients, so our conclusion still needs further verification and improvement.

\section{Conclusion}

In conclusion, we have come to realize that each cell in the body controls its growth and life status, usually through a network of molecular signals of environmental matter and energy supply. In this study, we first explored the relationship between the pathogenesis of IR in PCOS mice and PGC1 $\alpha$ molecules in ovarian tissue. Previous literatures were mainly focused on type 2 diabetes and metabolic disorders. These data enrich the current findings. Although there are some shortcomings, our work initially found that the insulin resistance-related AMPK pathway in the ovaries of PCOS mice is negatively correlated with the PGC $\alpha$ molecule. Since PGC1 $\alpha$ plays an important role in the metabolism of glucose and lipids metabolism, this result deserves further verification and exploration. PGC1 $\alpha$ may become the target of ovarian partial in the treatment of insulin resistance of women with PCOS in the future.

\section{References}

[1] Christensen, S.B., Black, M.H., Smith, N., Martinez M.M., Jacobsen, S.J., Porter, A.H. and Koebnick, C. (2013) The Prevalence of Polycystic Ovary Syndrome in Adolescents. Fertility and Sterility, 100, 470-477.

https://doi.org/10.1016/j.fertnstert.2013.04.001

[2] Fulghesu, A.M., Romualdi, D., Di Florio, C., Sanna, S., Tagliaferri, V., Gambineri, A., Tomassoni, F., Minerba, L., Pasquali, R. and Lanzone, A. (2012) Is There a Dose-Response Relationship of Metformin Treatment in Patients with Polycystic Ovary Syndrome? Results from a Multicentric Study. Human Reproduction, 27, 3057-3066. https://doi.org/10.1093/humrep/des262

[3] Liu, J., Zhu, Z.Y., Gao, Y.B., Geng, J.G., Zhao, X., Zhou S.N., Li, J.Y., Hong, Y. and Wang, X.L. (2016) Impacts of Tangzhiping on AMPK/PGC-1 $\alpha$ Signaling Pathway in the Rats of Insulin Resistance. World Journal of Integrated Traditional and Western Medicine, 8, 1087-1090.

[4] Zhou, Y., Lin, S., Zhang, L. and Li, Y. (2016) Resveratrol Prevents Renal Lipotoxicity in High-Fat Diet-Treated Mouse Model through Regulating PPAR- $\alpha$ Pathway. Molecular and Cellular Biochemistry, 411, 143-150. https://doi.org/10.1007/s11010-015-2576-y

[5] Yu, X., Zhang, L., Yang, X., Huang, H., Huang, Z., Shi, L., Zhang, H. and Du, G. (2012) Salvianolic Acid A Protects the Peripheral Nerve Function in Diabetic Rats through Regulation of the AMPK-PGC1 $\alpha$-Sirt3 Axis. Molecules, 17, 11216-11228. https://doi.org/10.3390/molecules170911216

[6] Tao, X., Chen, L., Cai, L., Ge, S. and Deng, X. (2017) Regulatory Effects of the AMPK $\alpha$-SIRT1 Molecular Pathway on Insulin Resistance in PCOS Mice: An in Vitro and in Vivo Study. Biochemical and Biophysical Research Communications, 
494, 615-620. https://doi.org/10.1016/j.bbrc.2017.09.154

[7] Cameron, J.L., Jain, R., Rais, M., White, A.E., Beer, T.M., Kievit, P., Winters-Stone, K., Messaoudi, I. and Varlamov, O. (2016) Perpetuating Effects of Androgen Deficiency on Insulin Resistance. International Journal of Obesity, 40, 1856-1863. https://doi.org/10.1038/ijo.2016.148

[8] Howitz, K.T., Bitterman, K.J., Cohen, H.Y., Lamming, D.W., Lavu, S., Wood, J.G., Zipkin, R.E., Chung, P., Kisielewski, A., Zhang, L.L., Scherer, B. and Sinclair, D.A. (2003) Small Molecule Activators of Sirtuins Extend Saccharomyces cerevisiae Lifespan. Nature, 425, 191-196. https://doi.org/10.1038/nature01960

[9] Patel, R. and Shah, G. (2017) Effect of Metformin on Clinical, Metabolic and Endocrine Outcomes in Women with Polycystic Ovary Syndrome: A Meta-Analysis of Randomized Controlled Trials. Current medical Research and Opinion, 33, 1545-1557. https://doi.org/10.1080/03007995.2017.1279597

[10] Sun, Y., Hong, J., Chen, M., Ke, Y., Zhao, S., Liu, W., Ma, Q., Shi, J., Zou, Y., Ning, T., Zhang, Z., Liu, R., Wang, J. and Ning, G. (2015) Ablation of Lgr4 Enhances Energy Adaptation in Skeletal Muscle via Activation of Ampk/Sirt1/Pgc1 $\alpha$ Pathway. Biochemical and Biophysical Research Communications, 464, 396-400. https://doi.org/10.1016/j.bbrc.2015.06.066

[11] Koves, T.R., Li, P., An, J., Akimoto, T., Slentz, D., Ilkayeva, O., Dohm, G.L., Yan, Z., Newgard, C.B. and Muoio, D.M. (2005) Peroxisome Proliferator-Activated Receptor-Gamma Co-Activator 1alpha-Mediated Metabolic Remodeling of Skeletal Myocytes Mimics Exercise Training and Reverses Lipid-Induced Mitochondrial Inefficiency. The Journal of Biological Chemistry, 280, 33588-33598. https://doi.org/10.1074/jbc.M507621200

[12] Jager, S., Handschin, C., St-Pierre, J. and Spiegelman, B.M. (2007) AMP-Activated Protein Kinase (AMPK) Action in Skeletal Muscle via Direct Phosphorylation of PGC-1alpha. Proceedings of the National Academy of Sciences of the United States of America, 104, 12017-12022. https://doi.org/10.1073/pnas.0705070104

[13] Chau, M.D.L., Gao, J., Yang, Q., Wu, Z. and Gromada, J. (2010) Fibroblast Growth Factor 21 Regulates Energy Metabolism by Activating the AMPK-SIRT1-PGC-1 $\alpha$ Pathway. Proceedings of the National Academy of Sciences of the United States of America, 107, 12553-12558. https://doi.org/10.1073/pnas.1006962107

[14] Kim, M.Y., Lim, J.H., Youn, H.H., Hong, Y.A., Yang, K.S., Park, H.S., Chung, S., Ko, S.H., Shin, S.J., Choi, B.S., Kim, H.W., Kim, Y.S., Lee, J.H., Chang, Y.S. and Park, C.W. (2013) Resveratrol Prevents Renal Lipotoxicity and Inhibits Mesangial Cell Glucotoxicity in a Manner Dependent on the AMPK-SIRT1-PGC1alpha Axis in db/db Mice. Diabetologia, 56, 204-217. | https://doi.org/10.1007/s00125-012-2747-2

[15] Stepto, N.K., Cassar, S., Joham, A.E., Hutchison, S.K., Harrison, C.L., Goldstein, R.F. and Teede, H.J. (2013) Women with Polycystic Ovary Syndrome Have Intrinsic Insulin Resistance on Euglycaemic-Hyperinsulaemic Clamp. Human Reproduction, 28, 777-784. https://doi.org/10.1093/humrep/des463 